\title{
COMPLEX SPACES WITH PLENTY OF STEIN SUBVARIETIES
}

\author{
GUNNAR BERG
}

\section{Introduction.}

As is well known, the closed subspaces of a Stein space are Stein. On the other hand, one realizes by considering a non-Stein domain in $\mathrm{C}^{2}$, and using the fact that non-compact one-dimensional complex spaces are Stein (cf. [4], p. 270), that one can not deduce the Steinness of a complex space from that of its closed, proper subspaces.

On the other hand, a domain in $C^{n}$ with the property that it intersects all hyperplanes in Stein domains is Stein, provided that the first Cousin problem is universally solvable on it, (cf. [3], p. 160).

It is shown here that we have a similar situation for complex spaces and manifolds, at least in the case when they are open subspaces and submanifolds of Stein spaces and Stein manifolds (Theorem 3 and Theorem 5).

The fact that we can deduce properties of a manifold from its proper, closed submanifolds hints at the possibility of an inductive result, and this is obtained in the form of a theorem on manifolds whose closed sub-manifolds are Cousin I (Theorem 6).

\section{An extension theorem.}

It is shown here, that in order to guarantee the extendability of holomorphic functions from certain proper closed Stein subspaces to the whole space, it is sufficient to assume that the first Cousin problem is universally solvable on the space, i.e. given an open cover $\left(U_{i}\right)_{i \in I}$ of the space, and meromorphic functions $f_{i} \in \mathscr{M}\left(U_{i}\right)$ such that $f_{i}-f_{j} \in \mathcal{O}\left(U_{i} \cap U_{j}\right)$, there is a meromorphic function $F$ on the whole space such that $F-f_{i} \in \mathcal{O}\left(U_{i}\right)$. If this is the case we say that the space is a Cousin I space.

The following result was first demonstrated in [1]. We repeat the easy proof.

Theorem 1. Let $X$ be a Cousin I space, and let $S$ be a Stein subspace of $X$, defined as the zero-set of a holomorphic function $f$ on $X$. Then every holomorphic function on $S$ can be extended to $X$.

Received March 24, 1981. 
Proof. Since $S$ is Stein, there is an open Stein neighbourhood $\Omega$ of $S$ in $X$ (see [5]). If now $g$ is holomorphic on $S$, then $g$ can, to begin with, be extended to $\Omega$, a classical result (cf. [4], p. 245).

Now put $U_{0}=\Omega, U_{1}=X \backslash S$ and $h_{0}=g / f, h_{1}=1$. Then $\left(U_{i}, h_{i}\right)$ defines an additive Cousin distribution on $X$, and hence there is a meromorphic function $F$ on $X$ such that the functions $g_{i}=F-h_{i}$ are holomorphic on $U_{i}, i=0,1$.

If we now put $G=F \cdot f$, we clearly have that $G$ is holomorphic on $X \backslash S$, and on $\Omega$ we have that $F=g_{0}+g / f$, so on this set $G=f g_{0}+g$, i.e. $G$ is holomorphic here too.

Furthermore, $G$ restricted to $S$ is equal to $g$ and hence $G$ provides the desired extension.

\section{Some results concerning Cousin I spaces.}

We will now use the extension theorem to deduce properties of a space from properties of its proper closed subspaces, and in order to do so we need a good supply of closed, globally defined subspaces. The easiest way to get this is to assume that the space is an open subspace of a Stein space.

This also has the advantage that we can use a well-known definition of domain of holomorphy (see below) which is easier to handle, than the more general ones involving envelopes of holomorphy (cf. [7]).

Definition. An open subspace $\Omega$ of a Stein space $X$ is a domain of holomorphy if there are no open subsets $U_{1}$ and $U_{2}$ of $X$ such that

i) $\varnothing \neq U_{1} \subset \Omega \cap U_{2}$,

ii) $U_{2}$ is connected and not contained in $\Omega$,

iii) for every holomorphic function $f$ on $\Omega$, there is a holomorphic function $F$ on $U_{2}$ such that $F=f$ on $U_{1}$.

This definition means, roughly speaking, that there is no part of the boundary of $\Omega$ over which all functions in $\mathcal{O}(\Omega)$ can be continued, not even to a many-sheeted domain over $X$.

We shall say that a closed subvariety of a complex space extends holomorphically to the space if all holomorphic functions on the sub-variety extend to the whole space.

THEOREM 2. Let the complex space $\Omega$ be an open subspace of the Stein space $X$, and assume that the intersection of $\Omega$ with every hypersurface in $X$, given as the zero-set of a non-constant holomorphic function on $X$, is a domain of holomorphy and extends holomorphically to $\Omega$. Then $\Omega$ is a domain of holomorphy. 
Proof. Let $U_{1}$ and $U_{2}$ be two open sets with the properties i) and ii) in the definition of domain of holomorphy above, and let $x \in U_{2} \cap b \Omega$.

If $f$ is a non-constant holomorphic function on $X$ such that $f(x)=0$, it may happen that the hypersurface $f^{-1}(0)$ does not enter $\Omega$ at $x$, but "bounces off" the boundary. However, if we consider a given neighbourhood of $x$, there is certainly one of the level sets $S_{\alpha}=\{z ; f(z)=\alpha\}$, where we may assume that $\alpha$ is small, with the property that there is a point $x_{\alpha} \in b\left(\Omega \cap S_{\alpha}\right)$ (relative to $S_{\alpha}$ ) which lies in the neighbourhood.

From the assumptions in the statement of the theorem it follows that $S_{\alpha} \cap \Omega$ is a domain of holomorphy (as an open subspace of the Stein space $S_{\alpha}$ ). Hence there is a holomorphic function on this set which cannot be continued over $x_{\alpha}$. The extendability assumption then gives a function on $\Omega$ with the same property. In fact, it is now evident that there is no function, holomorphic on $U_{2}$, which coincides with the extended function on an open subset of $U_{2} \cap \Omega$. Consequently, $\Omega$ is a domain of holomorphy and the theorem is proved.

If we now combine the above result with Theorem 1 and the fact that a Stein space is a domain of holomorphy, we get the following:

Theorem 3. Let $\Omega$ be an open Cousin I subspace of the Stein space $X$, and assume that the intersection of $\Omega$ with every hypersurface of $X$, given as the zeroset of a holomorphic function on $X$, is Stein. Then $\Omega$ is a domain of holomorphy.

As mentioned in the introduction all non-compact complex spaces of dimension 1 are Stein, and hence it follows that if $X$, and consequently also $\Omega$, have dimension 2, all hypersurfaces are Stein. This gives:

Corollary. An open Cousin I subspace of a 2-dimensional Stein space is a domain of holomorphy.

This result, first proved in [1], generalizes a result stated by H. Cartan in 1934 and proved by Behnke and Stein in 1937, which says that a Cousin I domain in $\mathrm{C}^{2}$ is a domain of homorphy.

\section{The manifold case.}

We now consider the case, when $\Omega$ is an open submanifold of a Stein manifold $X$. In order to obtain an analogue of Theorem 2, it is sufficient to consider non-singular hypersurface. In fact we have:

THEOREM 4. Let the complex manifold $\Omega$ be an open submanifold of a Stein 
manifold $X$, and assume that the intersection of $\Omega$ with every non-singular hypersurface in $X$, given as the zero-set of a non-constant holomorphic function on $X$, is a domain of holomorphy and extends holomorphically to $\Omega$. Then $\Omega$ is a Stein manifold.

Proof. Let us note first that there are plenty of non-singular, globally defined, hypersurfaces in $X$. In fact, a special case of Theorem 1.1 in [6] is that for every discrete set of points in $X$, there is a non-constant holomorphic function on $X$, whose zero-set is non-singular and contains the points.

So we can argue exactly as in the proof of Theorem 2 , and deduce that $\Omega$ is a domain of holomorphy.

Take a point $x \in b \Omega$ and a coordinate neighbourhood $U$ of $x$ with a biholomorphic map $\pi: U \rightarrow U^{\prime} \subset C^{n}$, and finally a Stein neighbourhood $W$ of $x$ in $U$. Then $\pi(\Omega \cap W)$ is a domain of holomorphy in $C^{n}$, and hence it is Stein.

So $\Omega \cap W$ is a Stein neig'. bourhood of $x$ and consequently every point on the boundary of $\Omega$ has a neighbourhood whose intersection with $\Omega$ is Stein, i.e. $\Omega$ is locally Stein.

According to a result of Docquier and Grauert [2], it follows that $\Omega$ is Stein, and we are done.

The analogue of Theorem 3 is an immediate consequence:

Theorem 5. Let $X$ be a Stein manifold, and let $\Omega$ be an open Cousin I submanifold of $X$ such that the intersection of $\Omega$ with every non-singular hypersurface in $X$, given as the zero-set of a holomorphic function on $X$, is a domain of holomorphy. Then $\Omega$ is Stein.

Proof. We only have to note that, as shown at the end of the proof of Theorem 4, the intersections of $\Omega$ with the hypersurfaces are in fact Stein. The theorem then follows as in the proof of Theorem 3.

Corollary. An open Cousin I submanifold of a 2-dimensional Stein manifold is Stein.

Theorem 5 and its Corollary hint at the possibility of using induction on the dimension of the manifold to get a stronger result and it will now be shown how this can be done.

Let us to illustrate start with the case $\operatorname{dim} X=3$, and let $\Omega$ be an open Cousin I subspace with the property that all non-singular hypersurfaces, given as the zero-sets of holomorphic function on $X$, intersects $\Omega$ in Cousin I manifolds. As closed submanifolds of the Stein manifold $X$ the above mentioned 
hypersurfaces are then 2-dimensional Stein manifolds, and since they are Cousin I it follows from the corollary above that they are Stein. Finally, applying Theorem 5 we deduce that $\Omega$ is Stein.

In general we have:

Theorem 6. Let $X$ be a Stein manifold and let $\Omega$ be an open Cousin I submanifold of $X$ with the property that all closed submanifolds of $\Omega$ are Cousin I. Then $\Omega$ is Stein.

Proof. Let $n$ be the dimension of $X$. For $n=2$ the result follows from the Corollary to Theorem 5 . Assume that the theorem is proved for all manifolds $X$ of dimension $\leqq n-1$.

Take a function $f$ which is non-constant and holomorphic on $X$, and consider the Stein subspace $S=f^{-1}(0)$ of $X$ which we can assume to be nonsingular. Then, from the assumptions made the set $\Omega^{\prime}=\Omega \cap S$ is Cousin I and an open submanifold of the Stein manifold $S$. Furthermore, all closed submanifolds of $\Omega^{\prime}$ are Cousin $\mathrm{I}$.

Since the dimension of $S$ is $n-1$ it follows from the induction hypothesis that $\Omega^{\prime}$ is Stein. A final appeal to Theorem 5 shows that $\Omega$ is Stein and we are done.

\title{
REFERENCES
}

1. G. Berg, On 2-dimensional Cousin 1-spaces, Math. Ann. 248 (1980), 247-248.

2. F. Docquier and H. Grauert, Levisches Problem und Rungescher Satz für Teilgebiete Steinscher Mannigfaltigkeiten, Math. Ann. 140 (1960), 94-123.

3. H. Grauert and R. Remmert, Theory of Stein spaces (Grundlehren Math. Wissensch. 236), Springer-Verlag, Berlin - Heidelberg - New York, 1979.

4. R. Gunning and H. Rossi, Analytic functions of several complex variables, Prentice-Hall, Inc., Englewood Cliffs, N.J., 1965.

5. Y.-T. Siu, Every Stein subvariety admits a Stein neighborhood, Invent. Math. 38 (1976), 89-100.

6. B. Strehl, Analytische Hyperflächen mit vorgegebenen Singularitäten, Math. Ann. 200 (1973), 165-173.

7. O. Suzuki, A new class of domains of holomorphy (I). Publ. Res. Inst. Math. Sci. 13 (1977), 497-521.

\author{
MATEMATISKA INSTITUTIONEN \\ UNIVERSITETET I UPPSALA \\ THUNBERGSV. 3 \\ 75238 UPPSALA \\ SWEDEN
}

